Appl. Set-Valued Anal. Optim. 3 (2021), No. 3, pp. 309-316

Available online at http://asvao.biemdas.com

https://doi.org/10.23952/asvao.3.2021.3.05

\title{
MINIMALITY CONDITIONS FOR CONVEX COMPOSITE FUNCTIONS AND AN APPLICATION IN VECTOR OPTIMIZATION
}

\author{
STEFAN HAMANN \\ Faculty of Natural Sciences II, Institute of Mathematics, \\ Martin Luther University Halle-Wittenberg, Halle (Saale), Germany
}

\begin{abstract}
We prove both necessary and sufficient conditions for weak sharp minima of convex composite functions, where the latter requires neither the (lower semi-)continuity of the convex function nor the restriction on Banach spaces, and permits an application to vector optimization in connection with the notion of super efficiency.
\end{abstract}

Keywords. Convex composite function; Minimality conditions; Super efficiency; Vector optimization; Weak sharp minimum.

\section{INTRODUCTION}

Let $X$ be a real normed space, $x \in A \subseteq X, h: X \longrightarrow \mathbb{R} \cup\{+\infty\}$ with $h(x) \neq+\infty$. Then we say that $h$ has a sharp minimum on $A$ at $x$ if there exist $r>0$, and $a>0$ such that

$$
h(v) \geq h(x)+a\|v-x\| \text { for all } v \in B(x, r) \cap A,
$$

where $B(x, r)$ denotes the open ball with center $x$ and radius $r$. This notion of minimality has been proven to be useful in obtaining minimality conditions for convex composite functions, i.e., functions $g \circ f$ with convex $g$ and continuous / differentiable $f([1,2])$. However, simple examples like the following show the need for a generalized concept that allows the possibility of multiple minima. Consider the (nonconvex) convex composite function $g \circ f$, where

$$
f: \mathbb{R}^{2} \longrightarrow \mathbb{R}^{3} \text {, defined by } f\left(p_{1}, p_{2}\right):=\left(p_{1},-p_{1},-p_{1} p_{2}\right),
$$

and

$$
g: \mathbb{R}^{3} \longrightarrow \mathbb{R} \text {, defined by } g\left(q_{1}, q_{2}, q_{3}\right):=\max \left\{q_{1}, q_{2}, q_{3}\right\} .
$$

It is easy to see that the origin can not be a sharp minimum of $g \circ f$ neither on $B^{1}:=\mathbb{R} \times \mathbb{R}_{+}$nor on $B^{2}:=\mathbb{R}^{2}$, whereas we have

$$
(g \circ f)\left(p^{i}\right)-(g \circ f)\left(y^{i}\right)=(g \circ f)\left(p^{i}\right) \geq\left|p_{1}{ }^{i}\right|=\boldsymbol{d}\left(p^{i}, C^{i}\right)
$$

for $i=1,2$ and all $y^{i}:=\left(y_{1}{ }^{i}, y_{2}{ }^{i}\right) \in C^{i}, p^{i}:=\left(p_{1}{ }^{i}, p_{2}{ }^{i}\right) \in B^{i}, C^{1}:=\{0\} \times \mathbb{R}_{+}$, and $C^{2}:=\{0\} \times$ $\mathbb{R}$, respectively. $(\boldsymbol{d}(\cdot, S)$ stands for the distance function associated to a subset $S$, defined by $\boldsymbol{d}(p$, $S):=\inf \{\|p-s\| \mid s \in S\}$ ). Thus $C^{1}$ and $C^{2}$ are sets of weak sharp minima in the sense of the definition below which is due to the concept introduced by Burke and Ferris [3].

E-mail address: stefan.hamann100@gmail.com.

Received February 10, 2021; Accepted September 3, 2021.

(C)2021 Applied Set-Valued Analysis and Optimization 
Definition. Let $B$ be a subset of a normed space $X, h: X \longrightarrow \mathbb{R} \cup\{+\infty\}, \emptyset \neq C \subseteq B \cap \operatorname{dom} h$ (where dom $h:=\{p \in X \mid h(p) \neq+\infty\}$ ) and $a>0$. Then, we say that $C$ is a set of weak sharp minima for $h$ on $B$ with modulus $a$ if

$$
h(v) \geq h(y)+a d(v, C) \quad \text { for all } v \in B, y \in C .
$$

The characterizations for weak minima, where the function $h$ is convex and lower semicontinuous, can be found in $[4,5]$. Burke and Ferris [6] studied the Gauss-Newton method for convex composite functions using the concept of weak sharp minima. So, it is of interest to obtain the conditions for weak sharp minima of convex composite functions. In Section 2, we investigate the characterizations for weak sharp minima within the framework of not necessarily complete spaces, and analogous to the basic characterization theorems in [4]. Closely related results in the context of error bounds can be found in a recent paper of Azé [7]. Section 3 provides an application of Theorem 2.2, which states a sufficient minimality condition, in vector optimization. This approach is quite different to the one followed in $[8,9]$, though it considers sharp minima of a scalarization function.

Throughout this paper, $X$ and $Y$ are real normed spaces, and $X^{*}$ and $Y^{*}$ are their topological duals, endowed with the dual norm, defined by $\left\|l^{*}\right\|:=\sup \left\{\left|l^{*}(z)\right| \mid\|z\| \leq 1\right\}$ for $l^{*} \in X^{*}\left(Y^{*}\right)$ and $z \in X(Y)$, respectively. Furthermore, $C$ is a closed convex subset of $X$ as well as

$$
g: Y \longrightarrow \mathbb{R} \cup\{+\infty\} \text { is a convex function and } f: X \longrightarrow Y .
$$

The closed unit balls of $X, Y, X^{*}$ and $Y^{*}$ are denoted by $B_{X}, B_{Y}, B_{X^{*}}$ and $B_{Y^{*}}$, respectively, and cor $S$ stands for the algebraic interior of a set $S$. A nonempty subset $K$ of $Y$ is said to be a cone if $t K \subseteq K$ for any $t \geq 0$ and a convex cone if, in addition, $K+K \subseteq K$. A cone is called pointed if $K \cap(-K)=\{0\}$.

As usual, we define

$$
\begin{gathered}
\partial g(y):=\left\{y^{*} \in Y^{*} \mid y^{*}(z-y) \leq g(z)-g(y) \text { for all } z \in Y\right\}, \\
\operatorname{dom} \partial g:=\{y \in \operatorname{dom} g \mid \partial g(y) \neq \emptyset\}, \\
\sigma_{M}\left(l^{*}\right):=\sup \left\{l^{*}(v) \mid v \in M\right\} \text { for } M \subseteq X, l^{*} \in X^{*}, \\
\sigma_{M}(z):=\sup \left\{l^{*}(z) \mid l^{*} \in M\right\} \text { for } M \subseteq X^{*}, z \in X \text { or } M \subseteq Y^{*}, z \in Y,
\end{gathered}
$$

$N(A, x):=\left\{v^{*} \in X^{*} \mid v^{*}(w-x) \leq 0\right.$ for all $\left.w \in A\right\}$ for $x \in A \subseteq X$, the normal cone to $A$ at $x$, $K^{*}:=\left\{y^{*} \in Y \mid y^{*}(k) \geq 0\right.$ for all $\left.k \in K\right\}$ for a convex cone $K \subseteq Y$, the dual cone of $K$,

$$
g_{+}^{\prime}(p, q):=\inf \left\{\frac{1}{t}(g(p+t q)-g(p)) \mid t>0\right\} \text { for } p \in \operatorname{dom} g, q \in Y,
$$

and

$$
M \circ l:=\left\{y^{*} \circ l \mid y^{*} \in M\right\} \text { for } l: X \longrightarrow Y, M \subseteq Y^{*}
$$

\section{CONDitions FOr WeAK Sharp Minima}

In this section, we establish a sufficient and a necessary condition for the existence of weak sharp minima of convex composite functions. Condition (2.4) in Theorem 2.3 is an adapted version of the basic Fermat rule in convex optimization, i.e., $0 \in \partial h(x)+N(S, x)$ for a convex function $h$ and a convex set $S$.

First, we recall a sufficient condition for sharp minima that is provided by the following result [1, Theorem 2.3]. 
Theorem 2.1. Let $A \subseteq X$, let f be Fréchet-differentiable at $x \in A$, and let $f(x) \in \operatorname{cor}(\operatorname{dom} g) \cap$ dom $\partial g$. If there exists $a>0$ such that

$$
\sigma_{\partial g(f(x))}\left(f^{\prime}(x)(w-x)\right) \geq a\|w-x\|=\sigma_{a B_{X^{*}}}(w-x) \text { for any } w \in A,
$$

then $g \circ$ f has a sharp minimum on A at $x$.

Inequality (2.1) leads us to a sufficient condition for weak sharp minima that is stated in the next theorem. Recall that $f$ is said to be Gâteaux-differentiable at $p \in X$ if there is some bounded linear functional $f^{\prime}(p): X \longrightarrow Y$ such that

$$
f^{\prime}(p)(h)=\lim _{t \rightarrow 0} \frac{1}{t}(f(p+t h)-f(p)) \quad \text { for any } h \in X .
$$

Definition. (cf. [10]) The function $f$ is said to be uniformly differentiable on $V \subseteq X$ if $f$ is Gâteaux-differentiable on $V$ and

$$
\forall \varepsilon>0 \exists \delta>0 \forall p, q \in V:\|p-q\|<\delta \Rightarrow\left\|f(p)-f(q)-f^{\prime}(q)(p-q)\right\|<\varepsilon\|p-q\| .
$$

Theorem 2.2. Suppose that $x \in C \subseteq A \subseteq X, f(x) \in \operatorname{dom} g$ and $g(f(x))=g(f(y))$ for any $y \in C$. Let $a>0, b>0, c>0$, and $r>0$ such that $f$ is uniformly differentiable on $B(x, r)$, $b B_{Y^{*}} \cap \partial g(f(y)) \neq \emptyset$ for any $y \in C \cap B(x, r)$ and

$$
\sigma_{b B_{Y^{*}}} \cap \partial g(f(y))\left(f^{\prime}(y)(w-y)\right) \geq \sigma_{a B_{X^{*}}} \cap N(C, y)(w-y) \quad \text { for all } w \in A, y \in C \cap B(x, r)
$$

as well as

$$
\forall \alpha>0, v \in A \backslash C \exists z \in C, L^{*} \in N(C, z) \cap B_{X^{*}}: L^{*}(v-z) \geq c\|v-z\|-\alpha .
$$

Then, there is a number $s>0$ with

$$
g(f(v)) \geq g(f(y))+\frac{a c}{2} \boldsymbol{d}(v, C)
$$

for all $v \in B:=\{u \in A \cap B(x, s) \mid \boldsymbol{d}(u, C) \leq c s\}$ and $y \in C$. In particular, $C \cap B(x, s)$ is a set of weak sharp minima for $g \circ$ f on $B \cap\{p \in X \mid \boldsymbol{d}(p, C)=\boldsymbol{d}(p, C \cap B(x, s))\}$ with modulus $\frac{a c}{2}$.

Proof. Choose $0<r_{0} \leq r$ such that

$$
\left\|f(p)-f(q)-f^{\prime}(q)(p-q)\right\| \leq \frac{a c}{2 b}\|p-q\|
$$

for all $p, q \in B\left(x, r_{0}\right)$. Now, take $v \in B \backslash C$ and $\left.\alpha \in\right] 0, c s\left[\right.$, where $s:=\frac{r_{0}}{3}$. From (2.3), one has $L^{*}(v-z) \geq c\|v-z\|-\alpha$ for some $z \in C, L^{*} \in N(C, z) \cap B_{X^{*}}$. Since

$$
\begin{aligned}
\boldsymbol{d}(v, C) & =\max \left\{l^{*}(v)-\sigma_{C}\left(l^{*}\right) \mid l^{*} \in B_{X^{*}}\right\}[11, \text { Theorem 3.8.2] } \\
& \geq \sup \left\{l^{*}(v-y) \mid y \in C, l^{*} \in B_{X^{*}} \cap N(C, y)\right\},
\end{aligned}
$$

it follows that

$$
\|v-z\| \leq \frac{1}{c}(L *(v-z)+\alpha) \leq \frac{1}{c}(\boldsymbol{d}(v, C)+\alpha)<s+s
$$

Hence

$$
\|x-z\| \leq\|x-v\|+\|v-z\|<s+2 s=r_{0} .
$$


For any $y \in C$, we have

$$
\begin{aligned}
& g(f(v))-g(f(y)) \\
& =g(f(v))-g(f(z)) \\
& \geq \sigma_{b B_{Y^{*}} \cap \partial g(f(z))}(f(v)-f(z)) \\
& \geq \sigma_{b B_{Y^{*}}} \cap \partial g(f(z))\left(f^{\prime}(z)(v-z)\right)-\sigma_{b B_{Y^{*}} \cap \partial g(f(z))}\left(f(z)-f(v)+f^{\prime}(z)(v-z)\right) \\
& \geq \sigma_{a B_{X^{*}}} \cap N(C, z)(v-z)-b \frac{a c}{2 b}\|v-z\| \\
& \geq a L^{*}(v-z)-\frac{a c}{2}\|v-z\| \\
& \geq a c\|v-z\|-a \alpha-\frac{a c}{2}\|v-z\| \\
& \geq \frac{a c}{2} d(v, C)-a \alpha .
\end{aligned}
$$

Since $\alpha$ is arbitrary in the interval ]0, cs[, we have the desired inequality.

Remark 2.1. Condition (2.3) holds for any Banach space $X$ and $c \in] 0,1[$ (see [12, Proposition 1.3]).

With regard to condition (2.2), we have the following result.

Proposition 2.1. Let $C \subseteq A \subseteq X$ (A convex). Let $f$ be be Gâteaux-differentiable on $C$, let $g$ be continuous, and let $\partial g$ be norm bounded on $f(C)$. Then, the condition (2.2) of Theorem 2.2 is fulfilled if

$$
(g \circ f)_{+}^{\prime}(y, v-y) \geq \operatorname{ad}\left(v-y, \operatorname{cl}\left(\mathbb{R}_{+}(C-y)\right)\right)
$$

for some $a>0$ and all $v \in A, y \in C$.

Proof. Let $b>0$ be chosen so that $\partial g(f(C)) \subseteq b B_{Y^{*}}$. Now apply a chain rule [13, Propositions 2.47 and 2.49] to $g \circ f$ : This function is directionally differentiable on $C$, and it follows

$$
\begin{aligned}
\sigma_{b B_{Y^{*}} \cap \partial g(f(y))}\left(f^{\prime}(y)(v-y)\right) & =\sigma_{\partial g(f(y))}\left(f^{\prime}(y)(v-y)\right) \\
& =g_{+}^{\prime}\left(f(y), f^{\prime}(y)(v-y)\right) \\
& =(g \circ f)_{+}^{\prime}(y, v-y) \\
& \geq a \boldsymbol{d}\left(v-y, \operatorname{cl}\left(\mathbb{R}_{+}(C-y)\right)\right) \\
& =\sigma_{a B_{X^{*}} \cap N(C, y)}(v-y),
\end{aligned}
$$

where $v \in A, y \in C$, due to $\partial \boldsymbol{d}\left(\cdot, \operatorname{cl}\left(\mathbb{R}_{+}(C-y)\right)\right)(0)=B_{X^{*}} \cap N(C, y)$.

Next, we give a necessary condition for weak sharp minima.

Theorem 2.3. Let $f$ be continuous at $x \in C \subseteq A \subseteq X$ (A convex), Gâteaux-differentiable on $C$ with $f^{\prime}$ bounded on $C \cap W$ (W a neighborhood of $\left.x\right)$. Let $g$ be continuous at $f(x) \in \operatorname{dom} g$, and let $a>0$ and $c>0$. If $C$ is a set of weak sharp minima for $g \circ f$ on $\{u \in A \mid \boldsymbol{d}(u, C) \leq c\}$ with modulus a, i.e., $g(f(w)) \geq g(f(y))+a \boldsymbol{d}(w, C)$ for all $w \in\{u \in A \mid \boldsymbol{d}(u, C) \leq c\}$ and $y \in C$, then there exist $d>0$ and $s>0$ such that

$$
a B_{X^{*}} \cap N(C, z) \subseteq d B_{X^{*}} \cap\left(\partial g(f(z)) \circ f^{\prime}(z)\right)+N(A, z)=\partial g(f(z)) \circ f^{\prime}(z)+N(A, z)
$$

for each $z \in B(x, s) \cap C$. 
Proof. Since $g$ is continuous at $f(x)$, we can find $s_{0}>0$, and $d_{0}>0$ such that

$$
\left|g\left(y_{0}\right)-g\left(y_{1}\right)\right| \leq d_{0}\left\|y_{0}-y_{1}\right\| \text { for all } y_{0}, y_{1} \in B\left(f(x), s_{0}\right) \text {. }
$$

Furthermore, there are $s_{1}>0, s_{2}>0, d_{1}>0$ with $f\left(B\left(x, s_{1}\right)\right) \subseteq B\left(f(x), s_{0}\right)$ and $\left\|f^{\prime}(y)\right\| \leq d_{1}$ for any $y \in B\left(x, s_{2}\right) \cap C$. Setting $s:=\min \left\{s_{1}, s_{2}\right\}$ and picking arbitrary $z \in B(x, s) \cap C, v \in A$, one has $z+t(v-z) \in\{u \in A \mid \boldsymbol{d}(u, C) \leq c\}$ for sufficiently small $t \in] 0$, 1]. Hence

$$
\frac{1}{t}(g(f(z+t(v-z)))-g(f(z))) \geq \frac{a}{t}(\boldsymbol{d}(z+t(v-z), C)-\boldsymbol{d}(z, C)) .
$$

Using [13, Propositions 2.47 and 2.49], we have

$$
\begin{aligned}
\sigma_{\partial g(f(z)) \circ f^{\prime}(z)}(v-z) & =g_{+}^{\prime}\left(f(z), f^{\prime}(z)(v-z)\right) \\
& =(g \circ f)_{+}^{\prime}(z, v-z) \\
& \geq a \boldsymbol{d}(\cdot, C)_{+}^{\prime}(z, v-z) \\
& =\sigma_{a B_{X^{*}}} \cap N(C, z)(v-z) .
\end{aligned}
$$

It follows from [2, Lemma 2.1] that

$$
a B_{X^{*}} \cap N(C, z) \subseteq \partial g(f(z)) \circ f^{\prime}(z)+N(A, z) .
$$

Moreover,

$$
\frac{1}{t}(g(f(z+t p))-g(f(z))) \leq \frac{d_{0}}{t}\|f(z+t p)-f(z)\|
$$

for any $p \in X$ and sufficiently small $t \in] 0,1]$. Hence,

$$
\sigma_{\partial g(f(z))}\left(f^{\prime}(z)(p)\right)=g_{+}^{\prime}\left(f(z), f^{\prime}(z)(p)\right) \leq d_{0}\left\|f^{\prime}(z)(p)\right\| \leq d_{0} d_{1}\|p\| .
$$

Thus (2.4) holds for $d:=d_{0} d_{1}$ due to $\partial g(f(z)) \circ f^{\prime}(z) \subseteq d B_{X^{*}}$.

Remark 2.2. Let the assumptions of Theorem 2.3 be fulfilled. Suppose that $X$ and $Y$ are Banach spaces, $f$ is continuously differentiable, and $f^{\prime}(x)$ is surjective. Then, one obtains condition (2.2). To see this, we apply [14, Lemma 3.6]. We can find $m>0$, and $r \in] 0, s]$ such that

$$
m B_{Y} \subseteq f^{\prime}(u)\left(B_{X}\right) \text { for any } u \in B(x, r),
$$

which implies the surjectivity of $f^{\prime}(u)$ for any $u \in B(x, r)$. Now, let $z \in B(x, r) \cap C, q \in Y$. There exists $p \in X$ such that $f^{\prime}(z)(p)=q$. It follows from (2.5) that

$$
\sigma_{\partial g(f(z))}(q)=\sigma_{\partial g(f(z))}\left(f^{\prime}(z)(p)\right) \leq d_{0}\left\|f^{\prime}(z)(p)\right\|=d_{0}\|q\| .
$$

Hence $\partial g(f(z)) \subseteq d_{0} B_{Y^{*}}$, which shows that (2.2) holds with $b=d_{0}$.

\section{An Application in Vector Otimization}

In the sequel, let $K \subseteq Y$ be a convex, closed, pointed cone, and consider the vector optimization problem

$$
\text { Minimize } f(v), v \in A \subseteq X \text {, with respect to } K,
$$

which consists in finding efficient points of $f(A)$ with respect to the ordering cone $K$. For any strict minimum $x$ of the scalarization function $d(f(\cdot)-f(x),-K)$ on $A, f(x)$ is an efficient point of $f(A)$ in the usual sense, i.e.,

$$
(f(A)-f(x)) \cap-K=\{0\} .
$$


If $C$ is a set of weak sharp minima for $d(f(\cdot)-f(y),-K)$ on $A$, where $y \in C$, we have at least

$$
(f(A \backslash C)-f(y)) \cap-K=\emptyset .
$$

It is therefore obvious to deal with this optimization problem by applying known results on sharp and weak sharp minima of convex composite functions, respectively.

Assume that $f$ is Fréchet-differentiable at $x \in A$ and $\mathbb{R}_{+}(A-x)$ has a weakly compact base. If for each $w \in A \backslash\{x\}$ there exists $l^{*} \in K^{*}$ with $l^{*}\left(f^{\prime}(x)(w-x)\right)>0$, then, due to [2, Theorem 3.1], $x$ is a sharp minimum of $\boldsymbol{d}(f(\cdot)-f(x),-K)$ on $A \cap B(x, r)$ for some $r>0$. Since

$$
\|f(v)-f(x)\| \leq\|v-x\|+\left\|f^{\prime}(x)(v-x)\right\| \leq\left(1+\left\|f^{\prime}(x)\right\|\right)\|v-x\|
$$

for $v \in B(x, s), r \geq s>0$ sufficiently small and therefore

$$
\boldsymbol{d}(f(v)-f(x),-K) \geq a\|v-x\| \geq \frac{a}{1+\left\|f^{\prime}(x)\right\|}\|f(v)-f(x)\|
$$

for $v \in A \cap B(x, s)$ and some $a>0$, by [15, Theorem 4.6], we have that $f(x)$ is a super efficient point of $f(A \cap B(x, s))$ in the sense of Borwein and Zhuang [16] and Zheng [17], i.e.,

$$
\forall \varepsilon>0 \exists \delta>0: \mathbb{R}_{+}(f(A \cap B(x, s))-f(x)) \cap(B(0, \delta)-K) \subseteq B(0, \varepsilon) .
$$

With regard to the notion of weak sharp minima, Theorem 2.2. allows us to prove the result stated below (note that $\partial \boldsymbol{d}(\cdot,-K)(Y) \subseteq B_{Y^{*}}$ for the application of this theorem).

Theorem 3.1. Let $f$ be uniformly continuous on $A \subseteq X, x \in C \subseteq A$ and $\boldsymbol{d}(f(x),-K)=$ $\boldsymbol{d}(f(y),-K)$ for any $y \in C$. Let $a>0, c>0, r>0$ such that $f$ is uniformly differentiable on $B(x, r)$ and

$$
\sigma_{\partial \boldsymbol{d}(\cdot,-K)(f(y))}\left(f^{\prime}(y)(w-y)\right) \geq \sigma_{a B_{X^{*}} \cap N(C, y)}(w-y) \quad \text { for all } w \in A, y \in C \cap B(x, r),
$$

as well as

$$
\forall \alpha>0, v \in A \backslash C \exists z \in C, L^{*} \in N(C, z) \cap B_{X^{*}}: L^{*}(v-z) \geq c\|v-z\|-\alpha .
$$

Then, there exists $s>0$ such that the following is true:

$\forall \varepsilon>0 \exists \delta>0 \forall v \in B: \exists y \in C(f(v)-f(y) \in B(0, \delta)-K) \Rightarrow \exists z \in C(f(v)-f(z) \in B(0, \varepsilon))$, where $B:=\{u \in A \cap B(x, s) \mid \boldsymbol{d}(u, C) \leq c s\}$.

Proof. From Theorem 2.2, there exists some $s>0$ with

$$
\boldsymbol{d}(f(v),-K) \geq \boldsymbol{d}(f(y),-K)+\frac{a c}{2} \boldsymbol{d}(v, C)
$$

for all $v \in B=\{u \in A \cap B(x, s) \mid \boldsymbol{d}(u, C) \leq c s\}, y \in C$. Now take an arbitrary $\varepsilon>0$ and choose $\gamma>0, \delta>0$ such that

$$
\|f(p)-f(q)\|<\varepsilon \quad \text { for } p, q \in A \text { with }\|p-q\|<\gamma
$$

and

$$
|\boldsymbol{d}(h,-K)|<\frac{a c \gamma}{4} \text { for }\|h\|<\delta .
$$

Consider

$$
f(v)-f(y)=h-k \in(f(B)-f(C)) \cap(B(0, \delta)-K),
$$


where $v \in B, y \in C, h \in B(0, \delta), k \in K$. We can pick $y^{*} \in \partial \boldsymbol{d}(\cdot,-K)(0)=K^{*} \cap B_{Y^{*}}$ such that $y^{*}(h-k)=\boldsymbol{d}(h-k,-K)$ as well as $z \in C$ with $\boldsymbol{d}(v, C) \geq\|v-z\|-\frac{\gamma}{2}$, and obtain

$$
\begin{aligned}
\frac{a c \gamma}{4} & >\boldsymbol{d}(h,-K) \\
& \geq y^{*}(h) \\
& \geq y^{*}(h-k) \\
& =\boldsymbol{d}(h-k,-K) \\
& =\boldsymbol{d}(f(v)-f(y),-K) \\
& \geq \boldsymbol{d}(f(v),-K)-\boldsymbol{d}(f(y),-K) \\
& \geq \frac{a c}{2} \boldsymbol{d}(v, C) \\
& \geq \frac{a c}{2}\left(\|v-z\|-\frac{\gamma}{2}\right),
\end{aligned}
$$

which implies $\|v-z\|<\gamma$ and thus $\|f(v)-f(z)\|<\varepsilon$.

\section{CONCLUSIONS}

This paper provides necessary and sufficient conditions for weak sharp minima of convex composite functions, defined on not necessarily complete normed spaces. An application of the sufficient condition in vector optimization is also given. However, it would be eligible to obtain sharper results than the one stated in Theorem 3.1 by using appropriate notions of efficiency. In view of the setting, future research should achieve results based on more general notions of (weak) sharp minima, for instance, within the framework of metric spaces.

\section{Acknowledgments}

The author would like to thank the referees for valuable comments which helped to improve the presentation of this paper.

\section{REFERENCES}

[1] S. Hamann, A sufficient minimality condition for convex composite functions, Far East J. Appl. Math. 97 (2017), 75-83.

[2] S. Hamann, Minimality conditions for convex composite functions and efficiency conditions in vector optimization, Appl. Set-Valued Anal. Optim. 1 (2019), 221-229.

[3] J. Burke, M. Ferris, Weak sharp minima in mathematical programming, SIAM J. Control Optim. 31 (1993), 1340-1359.

[4] J. Burke, S. Deng, Weak sharp minima revisited Part I: basic theory, Control and Cybernetics 31 (2002), 439-469.

[5] C. Li, L. Meng, L. Peng, J. C. Yao, Weak sharp minima for convex composite infinite optimization problems in normed linear spaces, SIAM J. Optim. 28 (2018), 1999-2021.

[6] J. Burke, M. Ferris, A Gauss-Newton method for convex composite optimization, Math. Program. 71 (1995), 179-194.

[7] D. Azé, Characterizations for existence of multipliers in mathematical programming in Banach spaces, Preprint, 2020.

[8] S. Xu, S.J. Li, Weak $\psi$-sharp minima in vector optimization problems, Fixed Point Theory Appl. 2010 (2010), 1-10. 
[9] M. Studniarski, Weak sharp minima in multiobjective optimization, Control and Cybernetics 36 (2007), 925 $-937$.

[10] R. Cilia, J.M. Gutiérrez, Factorization of weakly continuous differentiable mappings, Bull Braz. Math. Soc. 40 (2009), 371-380.

[11] C. Zalinescu, Convex Analysis in General Vector Spaces, World Scientific, Singapore, 2002.

[12] K.F. Ng, W.H. Yang, Error bounds for abstract linear inequality systems, SIAM J. Optim. 13 (2002), 24-43.

[13] J.F. Bonnans, A. Shapiro, Pertubation Analysis of Optimization Problems, Springer Science and Business Media, New York, 2000.

[14] X.Y. Zheng, K. F. Ng, Calmness for L-subsmooth multifunctions in Banach spaces, SIAM J. Optim. 19 (2009), 1648-1673.

[15] A. Zaffaroni, Degrees of efficiency and degrees of minimality, SIAM J. Control Optim. 42 (2003), 1071-1086.

[16] J.M. Borwein, D. Zhuang, Super efficiency in vector optimization, Trans. Amer. Math. Soc. 338 (1993), 105-122.

[17] X.Y. Zheng, Proper efficiency in locally convex topological vector spaces, J. Optim. Theory Appl. 94 (1997), 469-486. 